\title{
La légitimation des mesures d'exception dans la lutte antiterroriste en Europe
}

\section{Anastassia Tsoukala}

\section{(2) OpenEdition}

1 Journals

\section{Édition électronique}

URL : http://journals.openedition.org/conflits/2036

DOI : $10.4000 /$ conflits.2036

ISSN : $1777-5345$

Éditeur :

CCLS - Centre d'études sur les conflits lilberté et sécurité, L'Harmattan

\section{Édition imprimée}

Date de publication : 1 mars 2006

Pagination : 25-50

ISBN : 2-296-00622-1

ISSN : 1157-996X

\section{Référence électronique}

Anastassia Tsoukala, « La légitimation des mesures d'exception dans la lutte antiterroriste en Europe », Cultures \& Conflits [En ligne], 61 | printemps 2006, mis en ligne le 17 mai 2006, consulté le 30 mars 2021. URL : http://journals.openedition.org/conflits/2036 ; DOI : https://doi.org/10.4000/ conflits. 2036

Ce document a été généré automatiquement le 30 mars 2021.

Creative Commons License 


\title{
La légitimation des mesures d'exception dans la lutte antiterroriste en Europe
}

\author{
Anastassia Tsoukala
}

1 Si les attentats terroristes du 11 septembre 2001 ont marqué un tournant dans les politiques de sécurité européennes, ceci ne résulte guère de l'émergence d'une prétendue nouvelle menace, mais de leur impact sur le champ politique et le champ de la sécurité occidentaux. En effet, à part la nouvelle composition et l'extrême médiatisation d'éléments déjà intégrés dans le répertoire d'actions terroristes en Europe, l'actuelle menace du terrorisme ne saurait soulever de nouvelles questions quant à sa gestion puisque, même si nous admettons qu'elle provient en partie des réseaux internationaux, elle ne peut se préparer et se matérialiser qu'au niveau national, voire local. Elle relève donc d'un registre d'action familier qui, à son tour, renvoie à des stratégies et pratiques de sécurité ordinaires ou, tout au moins, appliquées depuis longtemps dans nombre de pays européens.

2 Habituellement occultée du débat public, cette non-nouveauté de la menace terroriste se trouve inversée dans les arguments des défenseurs de nouvelles politiques antiterroristes, qui justifient l'adoption de nouvelles mesures, parfois fort attentatoires aux libertés publiques, au nom justement de la protection des populations face à une menace « nouvelle » et particulièrement grave. L'étendue de cet article ne nous permet ni de montrer comment les politiques antiterroristes actuelles reproduisent la relation sécurité / libertés publiques observée lors des politiques antiterroristes antérieures ${ }^{1}, \mathrm{ni}$ d'expliquer en quoi elles accélèrent, complètent ou renforcent certains processus déjà mis en œuvre en matière de sécurité intérieure depuis la fin de la bipolaritée ${ }^{2}$ ni d'analyser leur rôle dans la reconfiguration du champ politique et du champ de la sécurité intérieure en Europe et outre-Atlantique.

3 Nous tâcherons, en revanche, de mettre en lumière les fondements du cadre conceptuel de ces nouvelles politiques en nous penchant sur les stratégies de légitimation adoptées en la matière par les principaux acteurs politiques européens. En effet, qu'on suive le 
modèle hégémonique gramscien ou l'analyse du pouvoir foucaldienne, la quête du consensus social reste toujours la norme dans le processus de légitimation du politique au sein des sociétés de type occidental. De ce point de vue, l'adoption de mesures d'exception dans le cadre de la lutte antiterroriste ne pouvait se faire en dehors d'une politique de communication visant justement à justifier le bien-fondé de ces mesures et, par conséquent, à écarter tout doute sur leur nécessité et sur leur impact néfaste sur l'Etat de droit et les libertés publiques. Cette politique de communication s'étant essentiellement appuyée sur les médias, l'analyse des stratégies de légitimation de nouveaux régimes juridiques d'exception passe par celle de la couverture médiatique des discours politiques relatifs à ces nouvelles mesures. Il importe, toutefois, de préciser que la mise en lumière de l'argumentaire des défenseurs des mesures d'exception n'impliquera aucune évaluation quelconque de l'impact de ces discours sur le public. Difficile, voire impossible à quantifier ${ }^{3}$, l'importance éventuelle de cet impact ne modifie aucunement les logiques et enjeux sous-tendant l'origine des discours en question et, in fine, ne nous apprend rien d'essentiel sur les intentions des acteurs impliqués dans cette politique.

La question de la légitimation des mesures d'exception sera abordée à travers l'analyse des déclarations faites à la presse dite de qualité par des politiciens, des experts en sécurité et des leaders d'opinion en France, en Grande-Bretagne et en Italie, entre le 11 septembre 2001 et le 11 juin $2003^{4}$. Soucieux de couvrir plusieurs tendances politiques, nous analyserons tous les articles publiés en la matière par les quotidiens nationaux suivants: Le Monde, Libération, Le Figaro, The Times, The Guardian, The Independent, La Stampa, La Repubblica.

5 A part les articles de La Repubblica, qui ont nécessité le dépouillement du journal, les articles ont été recueillis à travers la base de données électronique LexisNexis. Ont été identifiés et analysés 260 articles dans la presse britannique, 185 dans la presse française et 119 dans la presse italienne. Ces articles ont fait l'objet d'une analyse de contenu aussi bien quantitative que qualitative. Ont été prises en compte uniquement les déclarations citées entre guillemets; chaque déclaration a été comptée une seule fois, indépendamment du nombre de ses futures répétitions éventuelles par la presse. Bien que l'étendue des discours varie sensiblement d'un pays à l'autre, atteignant son sommet en Grande-Bretagne et son niveau le plus bas en Italie, l'analyse a révélé que les discours s'articulent essentiellement autour de deux positions: la défense des mesures d'exception et la quête d'équilibre. Pour des raisons pratiques, la présentation de ces positions sera illustrée par un nombre limité de citations, considérées comme représentatives en la matière.

La presse britannique

6 Dans la majorité des cas, les discours sur les mesures d'exception ont eu lieu lors des débats parlementaires sur l'adoption du Anti-Terrorism, Crime and Security Act 2001. L'une des dispositions les plus controversées de cette loi autorisait, par dérogation à l'art. 5(1) de la Convention européenne des droits de l'Homme, la détention sans procès d'étrangers résidant en Grande-Bretagne, s'ils étaient soupçonnés d'être liés à des milieux terroristes sans qu'ils puissent se faire expulser au nom de la protection des droits de l'Homme. Afin de pouvoir introduire cette mesure dérogatoire, le gouvernement britannique a dû déclarer un «état d'urgence menaçant la vie de la nation ». La défense des mesures d'exception 
7 Cette position a été surtout adoptée par le ministre de l'Intérieur, David Blunkett ${ }^{5}$, et, à un degré moindre, par le Premier ministre, Tony Blair ${ }^{6}$, et les leaders de l'opposition conservatrice, Oliver Letwin et Iain Duncan Smith.

Contrairement à ce que l'on pourrait attendre, l'importance et la nature extraordinaire de la menace terroriste constituent l'une des plus faibles bases de justification des mesures d'exception puisqu'elles ne sont évoquées que dans $13,7 \%$ des cas. Suivant cette série d'arguments, les mesures d'exception sont nécessaires à la protection de la Grande-Bretagne, confrontée à une menace imminente, grave et dotée d'une force de destruction considérable, une menace qui est "en hausse de manière dramatique, non seulement car les gens deviennent des kamikazes mais parce qu'ils ont déclaré la guerre à nous tous, dans le sens où ils s'organisent pour détruire nos vies, notre liberté et notre manière de vivre $^{8} \gg$.

9 Face à cette menace, le gouvernement est tenu de prendre toute mesure susceptible de garantir la sûreté publique et la sécurité nationale. Il n'est donc guère surprenant que les arguments les plus couramment évoqués (dans 31,3\% des cas) soient relatifs à cette responsabilité du gouvernement, laquelle est d'ailleurs souvent inscrite dans une logique de guerre. Puisque l'état de guerre est habituellement lié à des sacrifices, le fait de qualifier la lutte antiterroriste de guerre contre un nouvel ennemi ${ }^{9}$ permet à l'exécutif de présenter les mesures d'exception comme des sacrifices nécessaires à l'efficace protection de la population aussi bien en Grande-Bretagne qu'à l'étranger. Celles-ci sont alors présentées comme «essentielles pour combattre efficacement le terrorisme et nécessaires pour diminuer le risque d'un attentat terroriste dans ce pays et ailleurs ${ }^{10} »$. Une des principales caractéristiques de cet argumentaire est l'extension de la portée de la lutte antiterroriste vers l'ensemble du champ de la sécurité intérieure puisqu'il est clairement affirmé que le terrorisme est étroitement lié au crime organisé et à la hausse de l'insécurité en général : «les terroristes ne sont pas seulement impliqués dans des actes de terrorisme; ils sont aussi impliqués dans d'autres formes de criminalité afin de financer et de faciliter l'accomplissement de leur activités terroristes. La gestion policière du terrorisme passe alors par celle de ces crimes ${ }^{11}$ ". Partant du principe que ces activités criminelles interconnectées ne peuvent être combattues de manière efficace dans le cadre juridique en vigueur, les mesures d'exception sont présentées comme une mise à jour, urgente mais indispensable, de la loi en vigueur qui, loin de mettre en danger, vise à réaffirmer le règne de la justice en identifiant des « vides juridiques, des zones grises et des mesures existantes qui doivent être mises à jour en vue de contrôler les terroristes qui travaillent à travers les continents ${ }^{12}$ » de telle sorte que "la justice pour les particuliers et les minorités sera réaffirmée et que la justice pour la majorité et la sécurité de la nation sera assurée $^{13} »$.

10 On estime toutefois que la réalisation de cet objectif est mise en jeu par certaines contraintes posées par le cadre juridique en vigueur. Dans 17,6\% des cas, les défenseurs de cette thèse illustrent leurs propos en se référant à l'impact de la Human Rights Act 1998 sur le traitement réservé aux demandeurs d'asile. Bien établi dès le lendemain des attentats du 11 septembre, le lien entre terroristes et demandeurs d'asile devient de plus en plus présent dans les discours publics. Il est alors considéré qu'il est impossible de supporter une «situation où les gens arrivent, abusent de nos procédures de demande d'asile et ont quand même le droit d'y rester en demandant l'asile » ${ }^{14}$. Désormais, les termes « asile » et " abus » sont si étroitement liés que les mesures d'exception proposées par la loi deviennent la seule solution possible au problème. Par conséquent, on n'explique ni pourquoi les demandeurs 
d'asile seraient plus impliqués que les immigrés dans des activités terroristes, ni pourquoi un attentat terroriste serait exécuté par des étrangers plutôt que des ressortissants britanniques. En revanche, le ministre de l'Intérieur se sert souvent de métaphores simples, facilement compréhensibles, qui mettent en avant les concepts de la maison et de l'hospitalité. Mais, dans ses propos, l'hospitalité n'est pas comprise comme une responsabilité pour l'autre, suscitée par sa fragilité et souffrance ${ }^{15}$, mais comme une relation de pouvoir inégale, menant à des rapports de domination. Les demandeurs d'asile sont donc comparés à des invités ordinaires, que l'hôte peut mettre dehors à tout moment s'ils ne se comportent pas correctement : "Ceci est notre maison. C'est notre pays. Nous avons le droit de dire que si les gens abusent de la loi et cherchent à abuser du droit d'asile afin de pouvoir se cacher dans ce pays [...] nous devons prendre des mesures [pour les empêcher] ${ }^{16} »$. Lorsque la question des demandeurs d'asile se pose de nouveau, en 2003, le lien entre ceux-ci et le terrorisme est si solidement établi que les défenseurs de cette thèse peuvent demander de nouvelles mesures d'exception. Pour la première fois, ces mesures sont revendiquées aussi bien par le gouvernement que par l'opposition. Alors que le Premier ministre estime que, dans le contexte de la nouvelle menace terroriste, la « vague croissante » de demandeurs d'asile n'est plus acceptable, et déclare qu'il est prêt à reconsidérer "nos obligations sous le régime de la Convention des

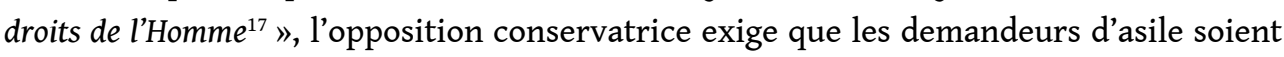
mis en détention jusqu'à ce que les services de sécurité aient établi qu'ils ne sont pas liés à des milieux terroristes. Ainsi, le Shadow Home Secretary ${ }^{18}$ demande-t-il que le pays "déroge à la Convention européenne des droits de l'Homme afin d'insister sur son droit d'expulser des faux demandeurs d'asile s'ils représentent une menace pour la sécurité nationale ${ }^{19}$ ». Partageant cet avis, le chef des Tories affirme que «des milliers de morts pourraient être provoqués par des terroristes présentés comme demandeurs d'asile " pour conclure qu'" un gouvernement Tory pourrait se retirer unilatéralement de la Convention de Genève si celle-ci n'était pas convenablement adaptée $e^{20}$ ".

L'acceptation de ces nouvelles mesures d'exception, attentatoires aux droits de l'Homme, n'est toutefois possible que si elle s'appuie sur une certaine reformulation du concept de la liberté et de la place détenue par les droits de l'Homme au sein de la société britannique. Ainsi, loin d'admettre que l'adoption d'un cadre juridique parallèle puisse mettre en jeu les libertés publiques, le ministre de l'Intérieur avance, dans 9,8\% des cas, l'idée que ces mesures sont indispensables même à la protection de la démocratie, nécessaires " pour garantir et sauvegarder notre manière de vivre face à ceux qui voudraient nous enlever notre libertée ", qui voudraient "détruire nos vies et notre démocratie $e^{22}$ ». Ces affirmations sont rendues possibles non seulement car les attentats du 11 septembre ont été qualifiés d'attaques contre la démocratie et la liberté, mais aussi parce qu'il est présumé que le droit des libertés publiques est destiné à la seule protection de la majorité. Les libertés publiques de la minorité sont ainsi sacrifiées au nom de la protection de la sécurité de la majoritée ${ }^{3}$. Par conséquent, le ministre de l'Intérieur peut déclarer: « Nous ne menaçons pas les libertés publiques de ce pays car nous ne parlons que d'une poignée d'individus, nous cherchons à nous assurer que cette poignée d'individus ne menace pas ces libertés publiques ${ }^{24} »$.

12 Les droits de l'Homme se trouvent alors transformés en un instrument politique, susceptible d'être appliqué de manière sélective. Désormais, ils peuvent faire l'objet de critiques, notamment à chaque fois que leur protection implique la restriction de l'exercice du pouvoir de l'exécutif. Il n'est donc guère surprenant que le ministre de l'Intérieur ait déclaré que l'incorporation de la Convention européenne des droits de 
l'Homme dans la loi britannique a été l'une des plus grandes erreurs du premier mandat du gouvernement Blair ${ }^{25}$.

La quête d'équilibre

13 Adoptée dans la plupart des cas par le ministre de l'Intérieur ${ }^{26}$ et, à un degré moindre, par les leaders de l'opposition conservatrice ${ }^{27}$, Oliver Letwin et Lord Strathclyde, cette position s'appuie sur l'idée que les droits de l'Homme constituent une valeur égale mais opposée à celle de la sécurité. Partant du principe que la protection de l'une ne peut se faire qu'aux dépens de l'autre, on présume que le gouvernement ne peut combattre efficacement la nouvelle menace terroriste que s'il crée un nouveau cadre juridique établissant un nouvel équilibre entre ces deux valeurs. Ce nouvel équilibre devrait être conforme au schéma juridique général selon lequel, en cas de conflit entre deux valeurs sociales également protégées par la loi, l'une doit être sacrifiée ou redéfinie pour qu'elle devienne subordonnée à l'autre. Les mesures prévues par la nouvelle loi sont alors présentées comme nécessaires à la protection des libertés publiques, car désormais «les libertés publiques doivent s'équilibrer vis-à-vis du besoin de maintenir l'ordre face à la menace [terroriste] ${ }^{28} »$. Le gouvernement doit alors trouver un nouvel équilibre entre «le respect de nos libertés publiques et la garantie que celles-ci ne sont pas exploitées ${ }^{29}$ ", car «le droit à la vie privée doit s'équilibrer vis-à-vis du droit à la sûreté et à la vie en liberté30."

$14 \mathrm{Au}$ lendemain des attentats du 11 septembre, l'idée d'un équilibre à trouver entre les valeurs, prétendument opposées, de la liberté et de la sécurité est partagée par tous les acteurs politiques impliqués dans le débat public. En effet, on admet généralement qu' i il faut bien trouver un équilibre entre la protection du citoyen et celle de la liberté31 » et il est rappelé que «le rôle historique du Parlement consiste à trouver le juste équilibre entre les besoins de sécurité et la protection de la libertés2 ". Néanmoins, vu que la restriction des libertés publiques au nom de la protection de la sécurité soulève inévitablement la question de la conformité de la nouvelle politique antiterroriste aux principes démocratiques fondamentaux, le gouvernement insiste lourdement sur les difficultés de cette tâche. Soucieux de montrer qu'il a conscience de l'importance des principes et valeurs en jeu et qu'il rejette tout modèle de pouvoir autoritaire et antidémocratique, il souligne qu'« arriver à trouver le juste équilibre entre notre vie privée et nos attentes de la part de l'Etat qu'il nous protège et facilite la jouissance de notre liberté est l'un des défis les plus difficiles auxquels nous nous sommes jamais confrontés ${ }^{33}$ ", mais que nous devons " relever si nous allons effectivement protéger la plus fondamentale de toutes les libertés, à savoir celle de vivre paisiblement sans peur ${ }^{34}$ ».

15 En 2003, l'idée d'un équilibre à trouver entre les valeurs, prétendument opposées, de la liberté et de la sécurité émerge de nouveau, au nom de l'efficacité de la lutte contre le terrorisme, mais cette fois-ci sa portée s'élargit afin de couvrir aussi les demandeurs d'asile. Le Shadow Home Secretary déclare alors que "nous avons besoin d'un nouveau régime, susceptible de concilier les intérêts nationaux avec les intérêts des vrais réfugiés et de garantir que le pays se charge de sa juste part de réfugiés, tout en nous permettant d'effectuer des contrôles efficaces de l'immigration en général ${ }^{35}$ ».

La presse française

16 Dans l'écrasante majorité des cas, les discours sur l'adoption de mesures d'exception ont eu lieu juste après les attentats du 11 septembre, lors des débats parlementaires sur l'adoption du projet de loi sur la sécurité quotidienne. Les dispositions les plus controversées de ce projet de loi portaient sur le renforcement des pouvoirs de police et l'accès des agents de sécurité publics aux données de communications téléphoniques et d'Internet. Ces discours sont toutefois restés fort limités suite à un accord politique 
passé entre le gouvernement et l'opposition, écartant tout recours devant le Conseil constitutionnel.

La défense des mesures d'exception

17 Cette position n'est adoptée par aucun acteur ou parti politique en particulier. Ses défenseurs viennent tant du gouvernement que de l'opposition et ne s'expriment qu'une seule fois. Bien que la faible étendue du débat public en France ne nous permette pas d'avoir des discours aussi variés que ceux observés dans le cas britannique, il nous est possible de discerner deux séries d'arguments.

18 Selon la première série, avancée dans $3,7 \%$ des cas, l'adoption de mesures d'exception se justifie par la gravité et la nature extraordinaire de la menace. L'avis du Président de la République selon lequel les dispositions de la nouvelle loi sont «proportionnelles à la gravité des risques ${ }^{36}$ » est ainsi partagé par des politiciens aussi bien socialistes que conservateurs, déclarant qu' «à période exceptionnelle, des mesures d'exception ${ }^{37}$ » et que le combat contre le terrorisme, à l'instar de «toute guerre, par nécessité, restreint les libertés démocratiques ${ }^{38}$ ». Mais, contrairement à ce qui a été observé dans le cas britannique, où l'origine de la menace était perçue comme essentiellement extérieure, en France la menace est perçue aussi bien comme extérieure qu'intérieure, puisqu'il est considéré que, indépendamment de leur nationalité, les jeunes des banlieues peuvent se trouver impliqués dans des activités terroristes. On affirme alors que «les mesures gouvernementales sont une bonne chose car la France est concernée [par la menace terroriste]. Nous sommes l'un des foyers de préparation du terrorisme. Ce n'est pas une politique sécuritaire, mais préventive ${ }^{39} »$. Tenant compte de la surreprésentation des populations des banlieues dans les statistiques de police sur certains types de délits, les défenseurs de cette thèse présument que les habitants de ces quartiers constituent une menace multiforme pour la sécurité du pays et devraient, par conséquent, être traités par la même loi : " la crise actuelle ne fait qu'exacerber l'insécurité quotidienne, c'est d'autant plus vrai que les deux sont liés, terrorisme et grand banditisme s'alimentant au terreau des

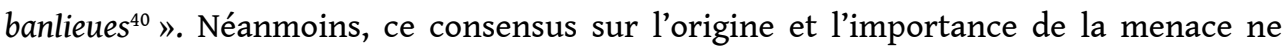
couvre pas également la durée des mesures d'exception. Alors que certains politiciens sont pour l'établissement d'un état d'exception permanent, estimant que «le combat contre le terrorisme est un combat de très longue haleine. Même si les mesures peuvent dans un premier temps être provisoires, pour des raisons juridiques, elles doivent à terme s'inscrire dans la duréé ${ }^{11} "$, d'autres jugent qu' «une fois la guerre terminée [on doit veiller] au rétablissement de toutes les libertés ${ }^{42} »$.

La deuxième série d'arguments, avancée dans 1,6\% des cas, est liée à la protection de la sûreté publique et de la sécurité nationale. Ces arguments s'appuient sur l'idée que "l'Etat de droit n'est pas l'Etat de faiblesse ${ }^{43}$ », car si la démocratie devient «synonyme de faiblesse, ce serait son arrêt de mort ${ }^{44} »$. Loin d'être considérées comme dangereuses pour la démocratie, les mesures d'exception sont présentées comme la preuve que la démocratie peut attaquer vigoureusement ses ennemis. Elles ne constituent pas un signe de déclin politique, mais sont, au contraire, l'expression d'une vitalité politique nécessaire à la protection de la démocratie et de la sécurité nationale. Il est ainsi affirmé que «c'est à l'Etat de mener la guerre antiterroriste. Sans aucune faiblesse, même s'il nous faut temporairement accepter des lois qui brident quelque peu nos libertés individuelles. Fouiller dans les coffres de voitures, c'est éviter que des lance-roquettes soient utilisées comme à Béziers; fouiller les sacs à main ou les personnes aux entrées des lieux publics, c'est éviter que des bombes ne soient déposées dans 
les grands magasins ou dans le métro comme en 1995 ; fouiller les appartements, c'est réduire les caches d'armes et les stocks de munitions ${ }^{45} »$.

Quoique extrêmes, ces mesures ne mettent pas en péril la démocratie, car elles visent à la protection de la sécurité collective, qui, en l'occurrence, «n'est pas l'ennemie de la liberté individuelle », puisqu'elle est «l'une des conditions d'exercice [de celle-ci] ${ }^{46} »$.

La quête d'équilibre

21 Cette position a été adoptée par la ministre de la Justice, Marylise Lebranchu, et le Bâtonnier de l'Ordre des avocats de Paris, Francis Teitgen. En général, elle s'appuie sur le schéma que nous avons déjà observé dans le cas britannique, à savoir la prétendue opposition entre la sécurité et les libertés publiques. Partant du principe que «les libertés individuelles et collectives [doivent] coexister avec le droit à la sûreté de tous et chacun ${ }^{47}$ ", on considère que la nouvelle menace a mis «la recherche d'un juste équilibre entre les exigences de la sécurité publique et la défense des libertés individuelles au coeur du débat d'aujourd'hui ${ }^{48} \gg$.

Toutefois, cette similitude concernant l'objectif à atteindre n'implique pas de similitude d'approche, le débat public français étant doté d'un aspect juridique qui fait défaut dans le cas britannique. Tout en précisant que «la liberté doit être placée avant la sécuritét ${ }^{49}$ ", Francis Teitgen suit la règle générale du sacrifice ou de la subordination d'une valeur sociale en conflit avec une autre et estime que la légitimité du sacrifice ou de la restriction dépend de la légalité des conditions déterminant le processus en question. Cette approche met, bien évidemment, l'accent sur l'intérêt collectif : «ces mesures ne sont justifiées que si le risque que l'exercice d'une liberté fait peser sur l'ensemble de la collectivité est tel que la mesure la plus extrême, seule, peut permettre de le conjurer "; dans ce cas, «la restriction à l'exercice d'un droit n'est acceptable que si elle est prévue par la loi, si elle est strictement nécessaire dans une société démocratique [...] et si elle strictement proportionnée à la menace qu'encourt l'ordre public ${ }^{50} »$.

La presse italienne

Contrairement aux deux cas précédents, la lecture de la presse italienne n'a pas révélé l'existence d'un débat public sur les mesures d'exception suite à l'adoption des articles 270(b) et (c) du Code Pénal sur l'association active au terrorisme, même international, ou la subversion de l'ordre démocratique ${ }^{51}$.

Cette absence de débat public pourrait résulter de deux facteurs distincts. D'une part, les nouvelles dispositions pénales ne faisaient qu'étendre au terrorisme le cadre juridique d'exception déjà en vigueur en matière de criminalité organisée. La longue acceptation de ce régime par la population et la classe politique a sans doute contribué à l'affaiblissement de toute réaction à l'extension de ce régime, dispensant de la sorte le gouvernement de toute justification publique. Cette absence de besoin de justification peut aussi être due au silence gardé en la matière par les partis de gauche qui, se trouvant dans une position inconfortable en raison de leur attitude vis-à-vis des activités terroristes de plusieurs groupuscules extrémistes de gauche dans les années 1970 et 1980, se montraient actuellement réticents à prendre une position qui pourrait être considérée comme favorable au terrorisme.

Conclusion

25 L'analyse des discours politiques sur la légitimation de nouvelles mesures d'exception dans les trois pays européens étudiés ici révèle trois tendances majeures, correspondant respectivement à l'émergence, aux spécificités et aux enjeux du débat public. D'abord, l'étendue du débat public est étroitement liée à l'existence ou non d'un 
effet de banalisation des procédures d'exception. Il semble pourtant que cette banalisation ne soit pas liée à la seule adoption des mesures d'exception, mais aussi au positionnement des acteurs politiques face à celles-ci. Ainsi, bien que la GrandeBretagne ait souvent connu, tout au long $\mathrm{du} \mathrm{xx}^{\mathrm{e}}$ siècle, des lois antiterroristes comportant des dispositions d'exception ${ }^{52}$, cette situation est toujours vécue comme extraordinaire par le monde politique. Par conséquent, à chaque fois que de telles lois sont mises en place, cela suscite un véritable débat public. Cette réaction du monde politique semble toutefois beaucoup moins forte en France, où le souci du maintien de certains équilibres au sein du champ politique semble l'emporter sur celui de la protection des libertés publiques, et atteint un niveau insignifiant en Italie, les acteurs politiques y étant soit bien familiarisés avec l'existence de mesures d'exception dans le cadre de la lutte contre la criminalité organisée, soit volontairement écartés du débat public afin d'éviter tout risque de ternir leur image.

Dans tous les cas de figure, dans les deux pays où il y a eu un débat public, la défense des mesures d'exception est certes largement couverte par la presse, car elle est habituellement adoptée par des acteurs politiques majeurs, mais ceux-ci ne représentent qu'une partie infime du monde politique. Par conséquent, la défense de cette thèse est qualitativement importante, mais reste quantitativement insignifiante et limitée à quelques individus. En revanche, la défense de la thèse intermédiaire, visant à la quête d'équilibre, jouit d'un support plus large, puisqu'elle est soutenue par un spectre plus vaste d'acteurs politiques et même par des membres de la société civile.

En outre, l'analyse révèle que, dans les deux pays où il y a eu un débat public, l'argumentaire utilisé reste sensiblement semblable. Ainsi, aussi bien en GrandeBretagne qu'en France, les défenseurs de l'adoption des mesures d'exception s'appuient notamment sur des arguments liés à la souveraineté, comme la protection de la sûreté publique et de la sécurité nationale. La seule divergence résulte de la perception dominante de l'origine de la menace. Perçue en Grande-Bretagne comme essentiellement extérieure au pays, celle-ci a contribué à l'alimentation des discours sur le renforcement du contrôle de l'immigration, alors qu'en France, son origine prétendument extérieure et intérieure au pays a donné lieu à des discours sur l'insécurité des banlieues. Les arguments avancés par les défenseurs de la quête d'équilibre semblent également être rapprochés, dans la mesure où, indépendamment de la place accordée au raisonnement juridique, ils partagent le même schéma oppositionnel de liberté/sécurité et admettent que la première valeur devrait être partiellement sacrifiée au profit de la seconde. Il est intéressant de noter que, bien que sous-tendant tous les discours favorables à l'adoption de mesures d'exception, la nouveauté de la menace, en tant qu'argument avancé de manière explicite, n'y occupe qu'une place secondaire.

Cette prépondérance des arguments liés à la souveraineté, qui n'alternent qu'avec des arguments inscrits dans le schéma oppositionnel précité, suggère toutefois que, au-delà du souci de rassurer la population sur la capacité de l'Etat à maintenir l'ordre et la sécurité à l'intérieur de ses frontières, les discours pour l'adoption de mesures d'exception visent également à permettre à leurs auteurs de se repositionner au sein de leur champ politique. Les résultats de l'analyse révèlent en fait que, en GrandeBretagne comme en France, la légitimation des mesures d'exception s'appuie in fine sur une modification de la notion de liberté et de droits de l'Homme. Ceci est bien révélé par le fait que les discours pour le droit d'exception mettent en avant une définition 
négative de la liberté. Celle-ci ne signifie plus liberté d'agir dans une société démocratique, mais liberté de jouir d'un ou plusieurs droits menacés. Par conséquent, les discours publics sur la liberté se transforment en discours sur la peur face à une menace présentée comme omniprésente et hautement imprévisible, contre laquelle il convient d'adopter toute mesure, même restrictive des libertés publiques, pourvu qu'elle soit considérée comme efficace. Loin de se référer aux libertés publiques, la liberté ainsi définie en vient alors à justifier la restriction même de celles-ci, laquelle devrait être acceptée, au nom de la protection de la liberté de ne pas avoir peur. Cette inversion des concepts, qui passe d'une définition positive de liberté d'action à une définition négative de liberté de ne pas avoir peur, est visible dans les discours aussi bien britanniques que français. Ainsi, alors que le ministre de l'Intérieur britannique déclare que les nouvelles mesures antiterroristes garantiront la protection de "la liberté de vivre en sécurité, sans peur, et bien évidemment sans risque de perdre sa vie ${ }^{53}$ ", son homologue français affirme que la sécurité ne s'oppose guère à la liberté individuelle mais, au contraire, qu'elle «est l'une des conditions d'exercice ${ }^{54}$ » de celle-ci.

Cette définition négative de la liberté va désormais de pair avec une restructuration de la place accordée aux droits de l'Homme dans les sociétés européennes. Il serait utile de rappeler ici que ces droits fondamentaux sont «le point de rencontre d'une conception déterminée de la morale sociale propre au monde moderne, une morale humaniste de la liberté et de l'égalité, et d'une conception politique également propre au monde moderne, qui est celle de la démocratie pluraliste ou de l'Etat démocratique de droit ${ }^{55} »$. Issu de la déclaration de 1948, ce grand mouvement universaliste a connu des débuts difficiles, mais a commencé à s'affirmer rapidement à partir des années 1970 de telle sorte que, tout en admettant que la protection des valeurs auxquelles se référait celui-ci pouvait parfois être relative, les gouvernements démocratiques ont fini par voir en ces droits une partie essentielle du cadre régissant l'existence même de la démocratie, au même titre que l'Etat de droit. On considérait par conséquent, que les limites ainsi posées à l'exercice de l'exécutif émanaient d'un consensus sur les valeurs que la communauté internationale acceptait comme fondamentales ${ }^{56}$. Inversement, il était clair que toute tentative de dépassement de ces limites nécessitait la mise en question de la nature de leur origine. Pourtant, selon les résultats de la présente analyse, le pouvoir exécutif a cherché, tant en Grande-Bretagne qu'en France, à élargir son champ d'action en niant aux droits fondamentaux ce rôle de cadre-régisseur du régime et en essayant de les traîner vers l'intérieur de ce cadre, en vue de les niveler avec les autres valeurs sociales mises sous la protection du gouvernement. En agissant ainsi, il fait voler en éclats leur contenu conceptuel fixe pour les transformer en contenants de concepts flexibles. Une fois abaissés au niveau de simples instruments politiques, ces droits peuvent s'ajuster aux besoins de chaque gouvernement. Par conséquent, ils peuvent être appliqués de manière sélective, faire l'objet d'interprétations variées, ou même être suspendus de manière provisoire sans que cela ne provoque de crises politiques majeures. Privés de l'essence de leur rôle politique, ils ne constituent plus un point de référence idéologique, intangible en démocratie, mais un outil de l'exercice du pouvoir, mis inévitablement au service de celui-ci. 


\section{NOTES}

1.. Della Porta D., «Institutional Responses to Terrorism : The Italian Case », Terrorism and Political Violence, 1992, 4, pp. 151-170 ; Schlagheck D., Walker J., « Democratizing Nations and Terrorism : The Effect of Political Violence on Civil Liberties », Current World Leaders, 1992, 2, pp. 287-309 ; Groenewold K., « The German Federal Republic's Response and Civil Liberties ", Terrorism and Political Violence, 1992, 4, pp. 136-150 ; Tsoukala A., "Les nouvelles politiques contre le crime organisé en Grèce », Revue de science criminelle et de droit pénal comparé, 1993, 3, pp. 603-608 ; Chalk P., « The Liberal Democratic Response to Terrorism », Terrorism and Political Violence, 1995, 4, pp. 10-44 ; Bossis M., Ellada kai tromokratia, Athens, Sakkoulas, 1996 ; Ewing K., Gearty A., The Struggle for Civil Liberties, Oxford/New York, Oxford University Press, 2000 ; Cettina N., L'Antiterrorisme en question, Paris, Michalon, 2001 ; Donohue L., Counter-terrorist Law and Emergency Powers in the United Kingdom 1922-2000, Dublin, Irish Academic Press, 2001. 2.. Waever O., "Securitization and Desecuritization », R. Lipshutz (dir.), On Security, New York, Columbia University Press, 1995, pp. 46-86 ; Beck U., Risk Society. Towards A New Modernity, London, Sage, 1996 ; Garland D., « The Limits of the Sovereign State. Strategies of Crime Control in Contemporary Society ", The British Journal of Criminology, 1996, 4, pp. 445-471 ; Ericson R.V., Haggerty K.D., Policing the Risk Society, Toronto, University of Toronto Press, 1997 ; Palidda S. (dir.), Immigrant Delinquency, Bruxelles, Commission européenne, 1997 ; Buzan B., Waever O., de Wilde J., Security : A New Framework for Analysis, Boulder, Lynne Riener, 1998 ; Jones R., « Digital Rule », Punishment \& Society, 2000, 1, pp. 5-22 ; Shearing C., «Punishment and the Changing Face of Governance », Punishment \& Society, 2001, 2, pp. 203-220 ; Mary P., Papatheodorou T., (dir.), Crime et insécurité en Europe, Bruxelles, Bruylant, 2001 ; Tsoukala A., «Le traitement médiatique de la criminalité étrangère en Europe ", Déviance et Société, 2002, 1, pp. 61-82 ; Ceyhan A., Tsoukala A., « The Securitization of Migration in Western Societies : Ambivalent Discourses and Policies ", Alternatives, 2002, 5, pp. 21-39 ; Silver E., Miller L., « A Cautionary Note on the Use of Actuarial Risk Assessment Tools for Social Control », Crime \& Delinquency, 2002, 1, pp. 138-161 ; Tsoukala A., « Les nouvelles politiques de contrôle du hooliganisme en Europe : de la fusion sécuritaire au multipositionnement de la menace ", Cultures \& Conflits, 2003, 51, pp. 83-96 ; Bigo D., « Les nouvelles formes de la gouvernementalité : surveiller et contrôler à distance », Ganjon M.-C. (dir.), Penser avec Foucault, Paris, CERI/Karthala, 2004, pp. 129-161; Bigo D., « Global (in)security : the field of the professionals of unease management and the Ban-opticon », Traces, 2004, 4 ; Huysmans J., « A Foucaultian View on Spill-over : Freedom and Security in the EU ", Journal of International Relations and Development, 2004, 7, pp. 294-318 ; Lyon D., « Globalizing Surveillance », International Sociology, 2004, 2, pp. 135-149 ; Tsoukala A., « Looking at Immigrants as Enemies », Bigo D., Guild E. (eds.), Controlling Frontiers : Free Movement into and within Europe, London, Ashgate, 2005, pp. 161-192 ; Stenson K., « Sovereignty, biopolitics and the local government of crime in Britain ", Theoretical Criminology, 2005, 3, pp. 265-287; Edwards A., Hughes G., "Comparing the Governance of Safety in Europe », Theoretical Criminology, 2005, 3, pp. 345-363.

3.. Jewkes, Y., Media \& Crime, London, Sage, 2004, p. 5 et suivantes. ; Maigret E., Sociologie de la communication et des medias, Paris, Armand Colin, 2004, p. 53 et suivantes. 
4.. Cette analyse s'inspire, en partie, de mon article « Democracy in the Light of Security ", paru dans Political Studies, 2006, 54(2), auquel le lecteur pourra se reporter, notamment s'il souhaite se renseigner sur les discours adoptés en la matière par les défenseurs des droits de l'Homme.

$5 . .37$ déclarations.

6.. 7 déclarations.

7.. 4 déclarations.

8.. Blunkett D., The Guardian, 20 novembre 2001.

9.. Voir, par exemple, la déclaration de Tony Blair sur « le nouveau type de guerre [contre le terrorisme]», The Guardian, 12 novembre 2002.

10.. Blair T., The Independent, 13 décembre 2001.

11.. Blunkett D., The Independent, 7 décembre 2001.

12.. Blunkett D., The Guardian, 20 novembre 2001.

13.. Blunkett D., The Times, 16 octobre 2001.

14.. Blair T., The Times, 1er octobre 2001.

15.. Venn C., « Altered States : Post-Enlightenment Cosmopolitanism and Trans-modern Socialities ", Theory, Culture \& Society, 2002, 1-2, p. 76.

16.. Blunkett D., The Independent, 20 novembre 2001.

17. Blair T., The Independent, 4 février 2003.

18.. Ministre de l'Intérieur fantôme.

19.. Letwin O., The Independent, 4 février 2003.

20.. Smith I. D., The Times, 29 janvier 2003.

21.. Blunkett D., The Guardian, 14 novembre 2001.

22.. Blunkett D., The Guardian, 20 novembre 2001.

23.. Waldron, J., « Security and Liberty : The Image of Balance », The Journal of Political Philosophy, 2003, 2, p. 194 et 203 et suivantes.

24.. Blunkett D., The Guardian, 14 novembre 2001.

25.. Blunkett D. The Independent, 12 novembre 2001.

26.. 8 déclarations.

27.. 3 déclarations.

28.. Blunkett D., The Independent, 12 novembre 2001.

29.. Blunkett D., The Independent, 16 octobre 2001.

30.. Blunkett D., The Guardian, 14 septembre 2002.

31.. Bingham R. (représentant de l'association de défense des droits de l'Homme Liberty) The Guardian, 29 septembre 2001.

32.. Lord Strathclyde, chef des Conservateurs dans la Chambre des Lords, The Independent, 6 décembre 2001.

33.. Blunkett D., The Guardian, 14 septembre 2001.

34.. Blunkett D., The Independent, 15 septembre 2001.

35.. Letwin O., The Independent, 29 January 2003.

36.. Chirac J., Libération, 17 novembre 2001.

37.. Touraine M., Galut Y., députés socialistes, Libération, 5 octobre 2001.

38. Debré B., ancien ministre conservateur, Le Figaro, 12 novembre 2001.

39.. Grzegrzulka O., député socialiste, Le Monde, 17 octobre 2001.

40.. Plasait B., sénateur « Républicains et indépendants », Le Monde, 1er novembre 2001.

41.. Lepeltier S., secrétaire général du RPR, Libération, 5 octobre 2001.

42.. Debré B., ancien ministre, Le Figaro, 12 novembre 2001.

43.. Badinter R., sénateur socialiste, Le Monde, 29 octobre 2001. 
44.. Debré B., Le Figaro, 12 novembre 2001.

45.. Debré B., Le Figaro, 12 novembre 2001.

46.. Vaillant D., ministre de l'Intérieur, Le Monde, 2 novembre 2001.

47. Teitgen F., président du Barreau de Paris, Le Figaro, 6 octobre 2001.

48. Lebranchu M., ministre de la Justice, Libération, 13 décembre 2001.

49.. Le Figaro, 6 octobre 2001.

50.. Teitgen F., Le Figaro, 6 octobre 2001.

51.. La seule déclaration en faveur de l'adoption d'un régime d'exception a été faite par le député européen Borghezio, proposant la fermeture des frontières pour les Musulmans (La Repubblica, 21 octobre 2001).

52.. Donohue L., Counter-terrorist Law and Emergency Powers in the United Kingdom 1922-2000, Dublin, Irish Academic Press, 2001 ; Ewing K., Gearty A., The Struggle for Civil Liberties, Oxford/New York, Oxford University Press, 2000.

53.. Blunkett D., The Times, 2 octobre 2001.

54.. Vaillant D., Le Monde, 2 novembre 2001.

55.. Peces-Barba G. « Les droits fondamentaux : entre morale et politique ", Pour les droits de l'Homme. Mélanges en l'honneur de l'ADLF, Choisy-le-Roi, Librairie des libertés, 1983, p. 95.

56.. Hoffman P., « Human Rights and Terrorism », Human Rights Quarterly, 2004, 4, p. 934.

\section{RÉSUMÉS}

Cet article vise à étudier la relation entre la sécurité et les libertés publiques, qui se trouve au cœur des débats sur les actuelles politiques antiterroristes en Europe. Il s'appuie sur une analyse des déclarations politiques faites par les défenseurs de l'introduction des mesures d'exception et leurs opposants, lesquelles ont paru dans la presse française, britannique et italienne, entre septembre 2001 et juin 2003. L'analyse révèle que : a) l'étendue du débat public est étroitement liée à l'existence ou non d'un effet de banalisation des procédures d'exception ; b) la légitimation des mesures d'exception s'appuie sur des arguments liés à la souveraineté qui, in fine, procèdent à une modification de la notion de liberté et de droits de l'Homme au sein des démocraties actuelles.

This paper aims to question the relation between security and liberty that lies at the heart of the current debates on European counterterrorism policies. It analyses the statements of the defenders of the emergency rules thesis and their rivals in the UK, France, and Italy which are reported in the press from September 2001 to June 2003. The findings reveal: a) that the extent of the public debate depends on the prior trivialisation of the emergency measures; $b$ ) that the legitimating of the emergency measures rests upon a set of sovereignty-related arguments that reframe the notion of freedom and the place of human rights in contemporary democracies. 
INDEX

Mots-clés : anti-terrorisme, exception

Index géographique : Europe

\section{AUTEUR}

\section{ANASTASSIA TSOUKALA}

Anastassia Tsoukala est maître de conférences à l'Université Paris-Sud XI, chercheur associé au Centre d'Etudes sur les Conflits et Senior Researcher dans les programmes ELISE et CHALLENGE. 